Review began 08/18/2021 Review ended 09/18/2021 Published 09/22/2021

\section{(๑) Copyright 2021}

Chotipanich. This is an open access article distributed under the terms of the Creative Commons Attribution License CC-BY 4.0., which permits unrestricted use, distribution, and reproduction in any medium, provided the original author and source are credited.

\title{
Total Laryngectomy: A Review of Surgical Techniques
}

\author{
Adit Chotipanich ${ }^{1}$ \\ 1. Otolaryngology Department, Chonburi Cancer Hospital, Ministry of Public Health, Chonburi, THA
}

Corresponding author: Adit Chotipanich,oat.adit@gmail.com

\begin{abstract}
Since the first total laryngectomy was performed in the late 18th century, several improvements and variations in surgical techniques have been proposed for this procedure. The surgical techniques employed in total laryngectomy have not been comprehensively discussed to date. Thus, the main objective of this article was to address controversial aspects related to this procedure and compare different surgical techniques used for a total laryngectomy procedure from the beginning to the end.
\end{abstract}

Although the management paradigms in laryngeal and hypopharyngeal squamous cell carcinomas have shifted to organ-preserving chemoradiotherapy protocols, total laryngectomy still plays a prominent role in the treatment of advanced and recurrent tumors. The increased incidence of complications associated with salvage total laryngectomy has driven efforts to improve the surgical techniques in various aspects of the operation. Loss of voice and impaired swallowing are the most difficult challenges to be overcome in laryngectomies, and the introduction of tracheoesophageal voice prostheses has made an enormous difference in postoperative rehabilitation and quality of life. Advancements in reconstruction techniques, tumor control, and metastatic management, such as prophylactic neck treatments and paratracheal nodal dissection (PTND), as well as the use of thyroid gland-preserving total laryngectomy in selected patients have all led to the increasing success of modern total laryngectomy. Several conclusions regarding the benchmarking of surgical techniques cannot be drawn. Issues regarding total laryngectomy are still open for discussion, and the technique will continue to require improvement in the near future.

Categories: Otolaryngology

Keywords: total laryngectomy, surgical technique, salvage laryngectomy, voice rehabilitation, neopharynx, pharyngeal fistula

\section{Introduction And Background}

The total laryngectomy procedure involves the removal of all laryngeal structures and a section of the upper trachea, which leads to disconnection of the airway and a permanent breathing hole in the neck (tracheostoma). In this approach, a cure for cancers is achieved at the expense of the patient's voice [1]. Total laryngectomy is also performed for non-cancerous conditions, such as severe trauma or chondronecrosis of the larynx. A reduction in the utilization of total laryngectomy has been observed $[2,3]$ since landmark trials in organ preservation for laryngeal squamous cell carcinoma by the Veterans Affairs Laryngeal Cancer Study Group in 1991 [4], followed by the Groupe d'Etude des Tumeurs de la Tête et du Cou group in 1998 [5] and the Radiation Therapy Oncology Group 91-11 in 2001 [6].

Based on the National Comprehensive Cancer Network Clinical Practice Guidelines in Oncology, total laryngectomy remains the standard treatment for T4a laryngeal squamous cell carcinoma and is a choice besides organ-preserving treatment for T3 laryngeal and T2-T4a hypopharyngeal squamous cell carcinomas [7]. With the exception of tumors with cartilage invasion, patients treated with organ-preserving treatment yield comparable oncologic outcomes to those treated with surgical modalities [8]. Organ-preserving treatment, if successful, preserves nearly normal laryngeal function, resulting in a better quality of life. However, patients with poor general health status or advanced age may not tolerate the toxicity of chemoradiation [4-6]. Organ preservation may not benefit patients with irreversible loss of laryngeal functions [9]. Approximately, one-fourth of patients undergoing organ-preserving treatment required salvage total laryngectomy because of a non-responsive tumor or complication associated with aspiration and necrosis [10]. The advantages and disadvantages of these treatments must be discussed with the patients to allow them to make an informed decision.

Recent decades have shown several changes in operative procedures. The surgical techniques for total laryngectomy have not been comprehensively discussed. This review aims to broadly summarize the variation and improvement in surgical technique for total laryngectomy. Since no previous review has addressed these topics within the scope of a single article, this review should help improve understanding of the surgical technique benchmarking and controversial aspects in each step of the operation.

\section{Review}




\section{Cureus}

\section{Incision}

Various incisions have been described for this procedure (Figure 1) [11,12]. A vertical midline incision provides direct access to the larynx but shows limited lateral exposure to the neck. T-shaped and double-Y incisions provide more exposure to the neck, but these incisions involve trifurcation, which results in a poor blood supply and inferior cosmetic results. Trap-door incisions preserve neck tissue for use in multi-stage reconstruction of extensive pharyngeal defects; however, their use has been abandoned in favor of contemporary single-stage reconstructions [12].

A
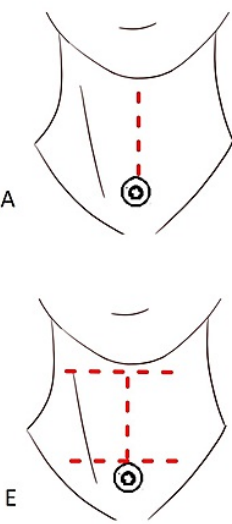
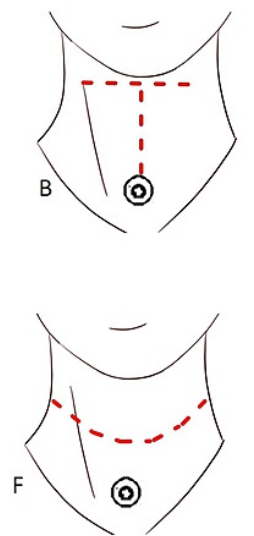
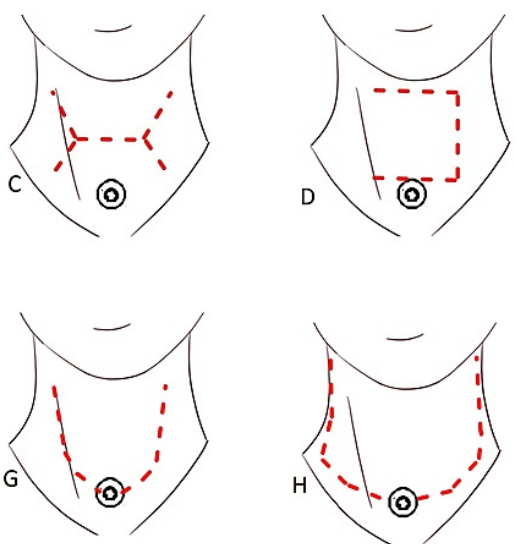

\section{FIGURE 1: Schematic illustration of the eight different types of neck} incisions.

(A) Midline vertical incision, (B) T-shaped incision, (C) horizontal double-Y incision, (D) trap-door incision, (E) double trap-doors, (F) apron incision (separate tracheostoma), (G) apron incision (tracheostoma-incorporated), and $(\mathrm{H})$ extended apron incision.

Apron incisions are considered the most favorable option for laryngectomy. A short apron incision involves a separate tracheostoma, and a long apron incision incorporates a tracheostoma with the incision. The short apron incision yields the best cosmetic result. The longer skin flap in the long apron incision may show decreased vascularity and increased venous and lymphatic congestion at the distal end. However, the short apron incision also resulted in restricted blood flow at a narrow skin portion between the incision and the tracheostoma.

The long apron incision involves a slightly higher incidence of peristomal dehiscence because the tension in this incision occurs directly to the tracheostoma [11]. The long apron incision provides good exposure to the lower-lateral neck. A larger size tracheostoma can be easily created, with the stoma sited within the main incision [13]. A long apron incision is a default option in patients showing lower-extension tumors or tumors requiring neck dissection.

\section{Management of paratracheal lymph nodes}

Paratracheal lymph nodes run along the sides of the trachea. Metastasis to these nodes can lead to peristomal recurrence and poorer clinical outcomes [14,15]. Paratracheal nodal dissection (PTND) may increase the risk of hypocalcemia and is not routinely performed in laryngectomy surgery [16].

Tumors involving the subglottic, pyriform sinus apex, and post-cricoid regions are at risk for paratracheal node metastasis $[17,18]$, and the rate of paratracheal nodal metastasis for tumors in these locations ranges between $12.5 \%$ and $51.1 \%$ [14-19]. Nodal dissection is the only way to reliably identify occult metastases and extranodal extension, which are crucial in predicting prognosis [20]. Prophylactic PTND shows improved local control and survival rates in salvage laryngectomy [15], but the benefit of adding prophylactic PTND in combined total laryngectomy and radiotherapy remains unclear [19].

Therapeutic PTND is necessary for patients with enlarged paratracheal lymph nodes. Prophylactic PTND should be performed in patients undergoing salvage total laryngectomy for recurrent T3-T4 tumors and is a considerable primary surgical option for tumors involving the subglottic, pyriform apex, and post-cricoid regions. Occult metastasis to contralateral paratracheal nodes is uncommon [18,19], and ipsilateral PTND in non-midline tumors can provide adequate results with minimal morbidity [21].

\section{Thyroid gland preservation}


The anatomical position of the thyroid gland renders it vulnerable to invasion by advanced laryngeal and hypopharyngeal cancers. In the past, ipsilateral hemithyroidectomy or total thyroidectomy was considered mandatory for all patients undergoing total laryngectomy [22]. However, several patients who underwent laryngectomies with partial preservation of the thyroid gland still developed hypothyroidism because the blood supply to the remnant thyroid gland was compromised during the operation [23]. Moreover, later studies have shown that thyroid gland invasion is uncommon [22-24]. Modern imaging techniques allow more accurate preoperative assessment of thyroid gland invasion. As a result, unnecessary thyroidectomy can be avoided and the incidence of postoperative hypothyroidism can be reduced [25].

Thyroid gland involvement in laryngeal and hypopharyngeal squamous cell carcinomas is mostly caused by direct extension. Lymphatic metastasis to the thyroid gland is rare, but it may occur because of the thyroid gland adjacent to the paratracheal lymph nodes [16-18]. Thyroid or cricoid cartilage invasion detected by preoperative imaging is a strong predictor of thyroid gland invasion [23,25]. Although uncommon, tumors may extend through the cricothyroid membrane or around the thyroid cartilage. These patterns of tumor invasion and lymphatic metastasis to the thyroid gland might not be detectable on CT images [23,25]. Routine thyroidectomy should help prevent missed diagnoses of thyroid gland involvement in tumors with a high risk of occult thyroid gland invasion and lymphatic metastasis.

The amount of thyroid gland tissue that needs to be removed depends on the extent of the tumor. Surgeons should be reminded that a positive surgical margin is associated with a worse prognosis. In cases showing gross invasion of the thyroid gland, total thyroidectomy is not an overtreatment. Hemithyroidectomy and/or isthmectomy are adequate when preoperative imaging shows only cartilage erosion without gross thyroid gland involvement. However, routine thyroidectomy is still recommended for tumors that involve the subglottic, post-cricoid, and pyriform sinus because of the high risk of occult thyroid gland involvement and lymphatic metastasis in these tumors. Apart from these concerns, thyroid-preserving laryngectomy in selected patients does not increase local recurrence rates, nor does it negatively affect disease-free survival [26].

\section{Management of neck metastases}

Comprehensive neck dissection (level I-V neck dissection) is generally considered the standard treatment for clinically positive neck nodes $(\mathrm{N}+)$ in head and neck cancers $[7,27]$. In laryngeal and hypopharyngeal squamous cell carcinomas, lymph node involvement is extremely rare at level I (0-2\%) and is rare at level V (0-6\%), even in cases of clinically $\mathrm{N}+$ [28-31]. A level II-IV select neck dissection (SND) can be alternatively performed in selected cases with limited nodal involvement (N1-N2a) [28].

In patients without clinically detectable lymph node enlargement (N0), prophylactic neck treatment is recommended for any $\mathrm{T}$ stage of supraglottic or hypopharyngeal squamous cell carcinoma, and T3/T4 glottic squamous cell carcinoma [7]. Bilateral neck treatment is required if the tumor exceeds the midline or presents with the craniocaudal extension of the larynx [31,32].

Elective neck dissection (END) is recommended as a prophylactic neck treatment in patients for which surgery is used as primary treatment [28,33]. END allows accurate pathological staging and determines whether adjuvant treatment is required $[7,34,35]$. For example, patients with T3N0 laryngeal squamous cell carcinoma can be safely managed with surgery alone if the pathological results from END show no occult lymph node involvement and other adverse features are not present [36].

Level II-IV SND is commonly used as END in total laryngectomy. Previous studies have shown that in a clinically N0 neck, lymph node level IIB has almost never been involved in supraglottic and glottic squamous cell carcinomas (0-1\%), and is rarely involved in hypopharyngeal squamous cell carcinoma (3\%) $[31,37-40]$. For lymph node level IV, occult lymph node involvement is rare to none in supraglottic and glottic squamous cell carcinomas $(0-3.5 \%)[33,41,42]$. To decrease postoperative morbidity as much as possible, super-SND at levels IIa and III in supraglottic and glottic squamous cell carcinoma has been advocated by several authors $[33,41,42]$.

\section{Removal of the larynx}

After the disinsertion of the strap and suprahyoid muscles and ligation of the feeding vessels, the larynx and trachea are dissected off the constrictor muscles and esophagus. The next crucial step is the removal of the larynx. This can be done using either a closed or an open technique.

The traditional technique requires entering the pharynx. The pharynx can be entered in the following locations: above the hyoid bone, lateral pharyngeal wall, and post-cricoid [43]. The pharynx should be entered at sites away from the tumor. A sufficient surgical margin is necessary for cancer surgery; however, there is no consensus on adequate safety margins in total laryngectomy [44]. In general, a margin of at least $5 \mathrm{~mm}$ seems acceptable in laryngeal and hypopharyngeal squamous cell carcinomas [45]. The disadvantages of opening the pharynx are salivary contamination and the need for manual closure. 


\section{Reconstruction of the neopharynx}

After removal of the larynx, the resulting defect of the pharynx is repaired, creating the so-called neopharynx. The ideal neopharynx must be watertight to avoid leakage, sufficiently large to allow food passage, and capable of accommodating voice rehabilitation.

The neopharynx must retain some degree of pharyngeal function to allow passage of food and production of alaryngeal speech. Loss of elasticity could result in a patulous passage, and conversely, excessive muscle tone can cause spasms; both conditions are undesirable for swallowing and speech rehabilitation [46,47].

In the closed technique, the larynx is separated from the pharynx by using a mechanical stapling device without entering the pharynx [48]. This closed technique significantly shortens the operation time and hospitalization [49]. Post-operative pharyngeal fistula and other complications occurring after stapling closure were shown to be comparable to, if not better than, those occurring after manual closure [48-52]. No study has confirmed the significant benefit of using stapling closure in salvage total laryngectomy [49]. The additional cost of using a stapling device must be considered against the benefits of the reduced operation.

In the closed technique, the lack of visualization of the tumor during resection is associated with the potential risk of inadequate surgical margins. Thus, the use of a stapler for pharyngeal closure should be performed only for tumors confined to the larynx $[49,50]$. In this regard, it may be safer to use the stapler closure technique for non-cancerous conditions.

Choosing Between Primary Closure or Other Reconstructions

Traditionally, the neopharynx is maintained wide enough to accept a 36-French bougie (approximately 3.8 $\mathrm{cm}$ in circumference). Hui et al. demonstrated that the narrowest width of the pharyngeal remnant (about $1.5 \mathrm{~cm}$ relaxed or $2.5 \mathrm{~cm}$ stretched) is sufficient both for primary closure and for restoration of swallowing function [53]. While these values are the theoretical lower limits of pharyngeal width that can be used in neopharyngeal reconstruction without significant stenosis, a larger neopharyngeal diameter does not correlate with better swallowing outcomes [53,54].

When achievable, primary closure is the first choice for neopharyngeal reconstruction because it has better function and is less complicated [55]. However, when primary closure is not possible, several reconstruction methods can be used. There is an ongoing controversy over the type of reconstruction that offers the best outcome.

In general, the choice of reconstruction is dictated partly by the expertise available and partly by the size of the defect. The commonly used reconstructions are pedicled flaps (e.g., pectoralis major myocutaneous flap) or free vascularized flaps (e.g., free radial forearm flap) in non-circumferential defects and gastric pull-up flaps in circumferential defects [56].

Management of salvage total laryngectomy with sufficient pharyngeal mucosa remains controversial. The fistular rates after salvage laryngectomy with primary closure vary from $10 \%$ to $78.6 \%$ [57-59]. A pooled analysis showed that primary closure combined with flap reinforcement using vascularized pedicled or free flaps reduced the risk of fistularity by one-third, compared to primary closure alone [60].

However, the routine use of flap reinforcement in salvage total laryngectomy has been a topic of controversy. Despite the scope for extended hospitalization and the potential need for further treatments [61], most fistulae are resolved using only conservative treatments [62]; therefore, routine flap reinforcement may add unnecessary morbidities associated with additional flap harvesting. Thus, vascularized flap reinforcement may be beneficial in patients with considerable post-radiation effects and should be considered on a case-by-case basis.

The Mucosal Layer

The mucosal layer of the neopharynx must be repaired to create a watertight closure. The mucosal repair can be either straight (vertical or horizontal) or a T-shaped line. The choice is based on the surgeon's preference and defect shape. Small defects are usually repaired using straight lines, while in larger defects, mucosal repair often results in a T-shaped line (Figure 2). 


\section{Cureus}

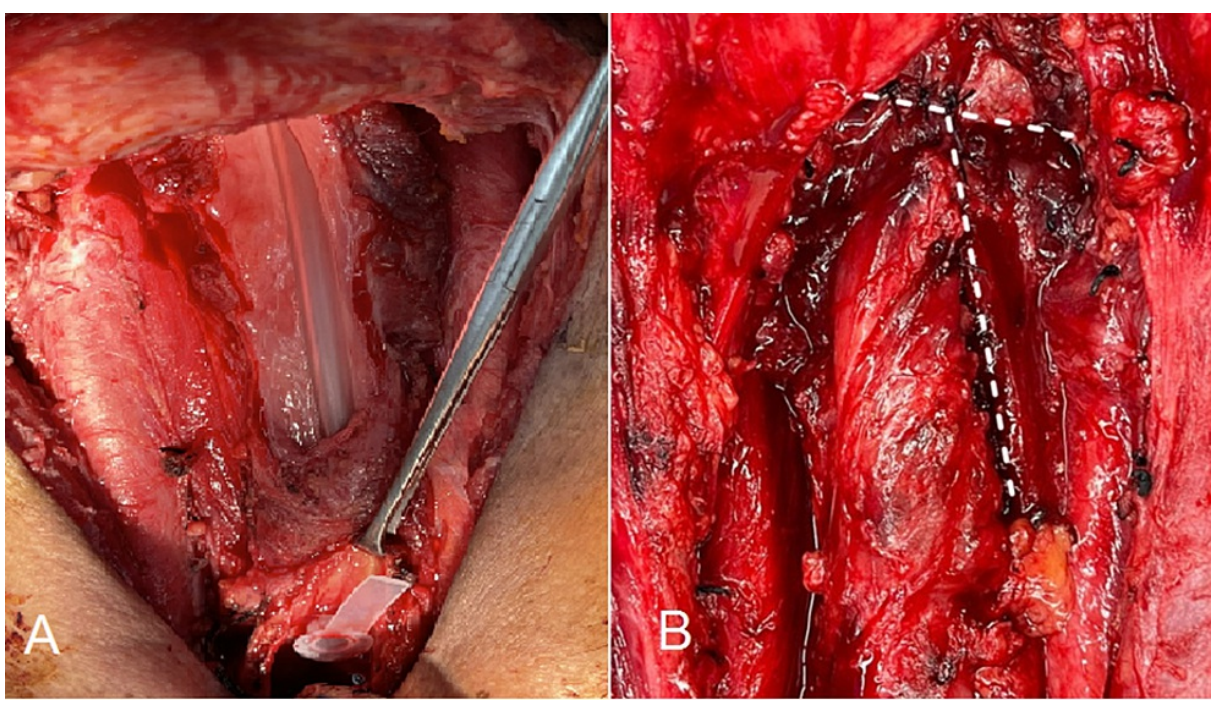

FIGURE 2: The mucosal layer of the neopharynx.

(A) The defect after total laryngectomy with partial hypopharyngectomy and (B) the T-shaped closure.

Caution should be exercised when applying the vertical line closure since surplus tissue at the midline of the neopharynx can create a pseudo-diverticulum [63], which can cause postoperative dysphagia [64,65]. The pseudo-diverticulum occurs less often when applying the T-shaped closure (84.6\% in vertical closure and $18.5 \%$ in T-shaped closure) [63]. The swallowing function of horizontal and T-shaped closures was found to be superior to that of vertical closure $[63,64]$.

In theory, the trifurcation in the T-shaped closure might increase the risk of fistula development, which is supported by the findings of some studies [66,67]. However, in contrast, other studies have suggested that a fistula occurs more frequently with vertical closure $[63,68,69]$. It is assumed that the T-shaped closure causes less tension than the vertical closure in some defect shapes. The horizontal closure appears to be the ideal closure line because it avoids trifurcation and produces a relaxed neopharynx with improved swallowing function $[64,70,71]$. However, horizontal closure may not be suitable for vertically extended pharyngeal defects.

The suturing technique for mucosal closure is critical because it must ensure that the mucosa is properly inverted and closed without excessive tension. Numerous techniques are available and can be broadly classified into interrupted and continuous sutures (Figure 3). Continuous sutures have the advantage of evenly distributing the wound tension. However, if a knot breaks, wound dehiscence can occur easily. In this respect, interrupted sutures provide better security. Recent studies have shown that the use of continuous sutures is associated with lower fistula rates than interrupted sutures in mucosal repair $[67,72,73]$. 


\section{Cureus}

A

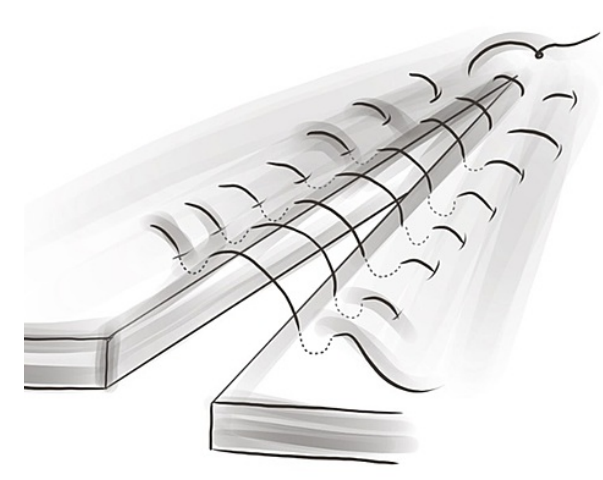

B

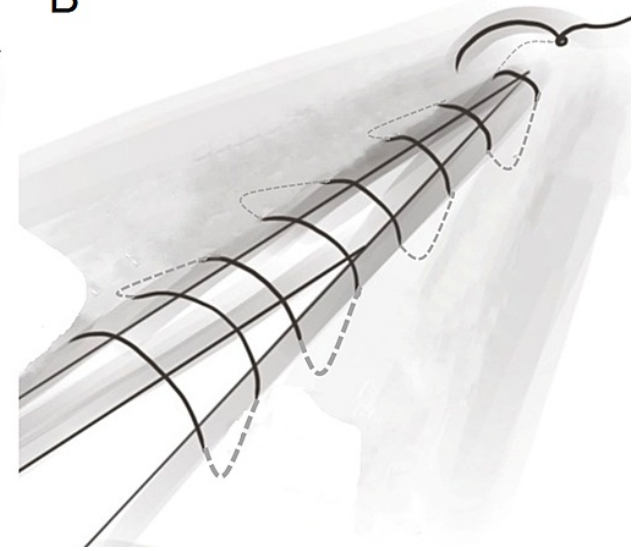

FIGURE 3: Schematic illustration of commonly used suture patterns in mucosal layer repair.

(A) The Lambert and Gambee interrupted-suture techniques and (B) the Cushing and Connell continuous-suture techniques. The Cushing and Lambert techniques penetrate only the submucosa, while the Connell and Gambee techniques pass through the lumen.

\section{The Submucosal and Muscular Layers}

In the traditional technique, the pharyngeal defect is closed in three layers (mucosal, submucosal, and muscular layers; Figure 4A). However, $12 \%-35 \%$ of patients who underwent the traditional three-layer closure failed to achieve satisfactory speech after tracheoesophageal puncture because of cricopharyngeal spasms $[74,75]$.

The exact mechanism underlying these spasms remains unclear. Surgery may damage the branches of the vagus nerve, resulting in uncoordinated contractions of the pharyngeal constrictor muscles [76]. To avoid spasms, later studies suggested adding either pharyngoesophageal myotomy (Figure $4 B$ ) or unilateral pharyngeal plexus neurectomy [77,78]. The advantage of neurectomy is that the vascularity of the pharyngeal wall is not compromised. However, spasms may still occur after the neurectomy because the cricopharyngeal muscle can be innervated from the recurrent laryngeal nerve or the contralateral pharyngeal plexus [79]. Thus, the combination of neurectomy and myotomy may be superior to either technique alone in cricopharyngeal spasm prevention [75]. A study comparing combined myotomy-neurectomy to pharyngeal myotomy alone found a lower incidence of cricopharyngeal spasm in the combined myotomy-neurectomy group, but that study did not show a significant difference in speech outcomes between these two groups $[75,80]$.

Another modified method for preventing cricopharyngeal spasm is to avoid complete circumferential repair of the pharyngeal musculature (Figure $4 C-4 F$ ) [71]. Non-closure, half-muscle closure, horizontal closure, and crossover zigzag neopharyngoplasty were superior to the traditional three-layer closure. One study also suggests that these modified methods may be performed in combination with pharyngoesophageal myotomy to minimize the intra-luminal pressure of the neopharynx which may lead to an improved voice outcome [80]. 


\section{Cureus}

A

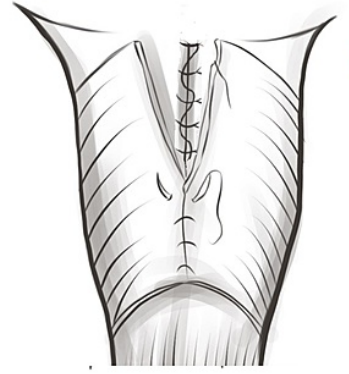

B
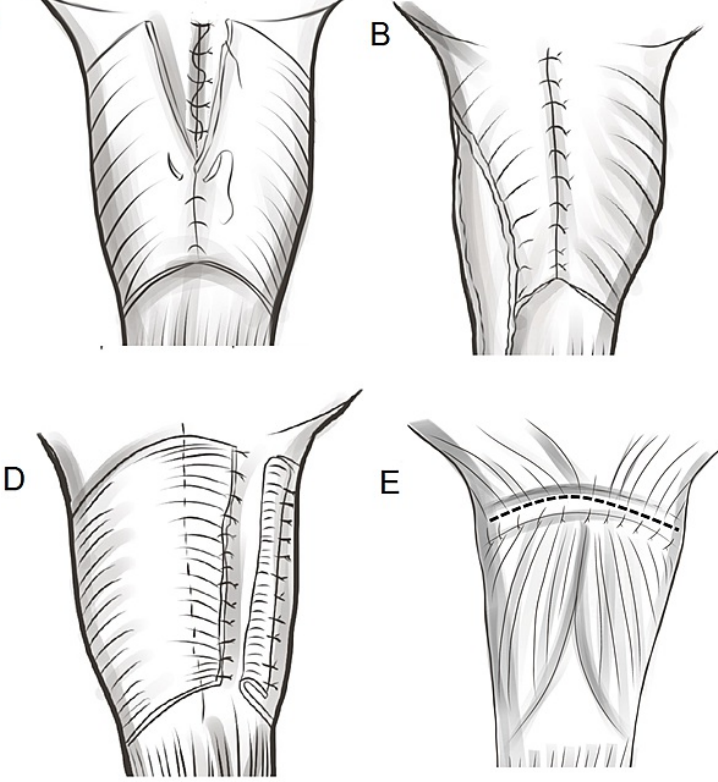

E

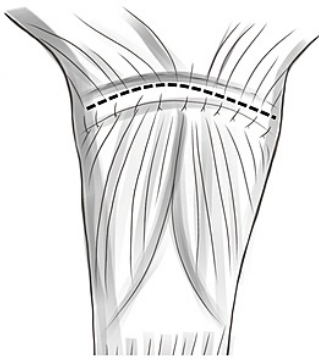

C
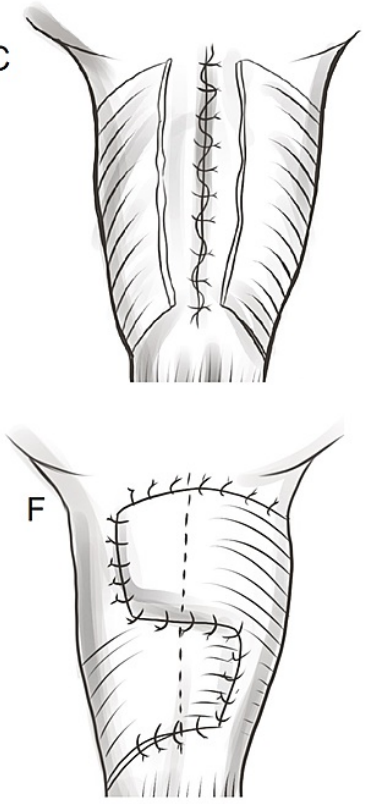

FIGURE 4: Schematic illustration of the reconstruction of the neopharynx.

(A) Traditional three-layer closure, (B) three-layer closure with myotomy, (C) non-closure of the pharyngeal musculature, (D) half-muscle closure technique, (E) horizontal closure, and (F) crossover zigzag neopharyngoplasty. In horizontal closure, the pharyngeal constrictors are stitched to the tongue base muscles.

Currently, these additional or modified techniques have shown more success in voice restoration than the traditional three-layer closure, and are associated with lower rates of voice restoration failure (between $0 \%$ and 10\%) [77,79-84]. Based on the literature, pharyngoesophageal myotomy is the most commonly used technique $[74-77,79,80,83,84]$. However, there is still no consensus on the superiority of any of these additional or modified techniques because of the lack of standard assessments of voice quality and wellcontrolled studies.

\section{Surgical voice rehabilitation}

Currently, there are three methods of voice rehabilitation after total laryngectomy: esophageal speech, electrolarynx, and surgical voice rehabilitation. Esophageal speech is achieved through a process of esophageal insufflation with swallowed air. The air is released from the esophagus, making the juncture of the neopharynx and esophagus a vibratory sound source for alaryngeal speech. Esophageal speech is more difficult to learn than the other two methods [85].

The electrolarynx produces voice using a device to emit constant vibrations that are transmitted to the pharynx through cervical skin or directly to the intraoral mucosa. The electrolarynx does not require surgical intervention or suitable neopharyngeal function. However, current commercially available electrolarynxes provide inferior voice quality compared to the other methods [85].

The idea of surgical voice rehabilitation after total laryngectomy is to create a connection between the trachea and the esophagus, which shunts pulmonary air to the neopharynx and esophagus juncture. The tracheoesophageal connection needs to be stable enough to prevent spontaneous closure while simultaneously allowing air to be easily drawn into the esophagus and preventing the aspiration of saliva [86].

Previously, tracheoesophageal connections were created using the patient's own tissues. Various techniques with moderate success rates have been described for this purpose, but all of them had high complication rates, mainly due to shunt breakdown and aspiration. Because of these and the complexity of some of the procedures, these techniques have been abandoned since a tracheoesophageal voice prosthesis (TEP) was invented [87].

Currently, TEP is considered the gold standard for voice rehabilitation. TEP uses a one-way valve to push air up from the lungs to pass through from the trachea and enter the esophagus without letting food or liquids pass through the other way. Patients simply occlude a stoma with a finger or a hand-free valve. The surgical 


\section{Cureus}

process is simple, with the only insertion of a TEP through a tracheoesophageal puncture behind the tracheostoma. A tracheoesophageal puncture can be performed at the time of laryngectomy (primary puncture) or at a later date (secondary puncture) [88].

During larynx removal, care should be taken to keep the tracheoesophageal party wall intact at the puncture site. Separation of this wall could cause a postoperative fistula. Primary TEP is considered to be associated with an increased risk of surgical complications, such as infection, stoma stenosis, fistula, and leakage $[89,90]$. However, these complications are infrequent and usually not severe. Moreover, there is no robust evidence to suggest that primary TEP is associated with poorer outcomes than secondary TEP [89].

Apart from the reasons already mentioned, primary TEP should be preferred because it avoids a second surgical intervention and allows early voice restoration, thereby exerting a positive psychological impact on patients who have undergone total laryngectomy.

\section{Tracheostoma creation}

The final step of the operation is the creation of a tracheostoma. The skin and the tracheal opening are sutured together using half-mattress sutures, which pull the skin over the exposed trachea ring. The diameter of the stoma should be more than $14 \mathrm{~mm}$ to ensure an adequate airway [91]. However, a stoma that is too large makes speech using stoma occlusion difficult; thus, the stoma should not be larger than the patient's thumb.

Stenosis of post-laryngectomy tracheostoma is common. The stomal construction is an important determinant of stomal stenosis. Constricting scars and stenoses occur when the trachea is insufficiently anchored to the skin and when raw areas are left at the junction between the skin and the tracheal mucosa. Diabetes mellitus and related tracheostoma infection are also considered risk factors for tracheostoma stenosis [92].

A simple technique to avoid tracheostoma stenosis is to create a larger stoma. A straight transection of the trachea (Figure $5 \mathrm{~A}$ ) results in a smaller diameter and a higher stenosis rate compared to the beveled technique (Figure $5 B$ ) [93]. The stoma can be created with a much greater diameter when the tracheal opening is extended to the lower neck by four or five tracheal rings, forming a triangular shape (Figure $5 \mathrm{C}$ ) [94]. The triangular stoma technique fully prevents stenosis, but its size and shape are troublesome for speech using stoma occlusion.

Another technique is the use of a skin flap interposed in the tracheal opening to prevent circular scars $[93,95]$. This technique, which can be performed with various designs (Figure $5 D-5 E$ ), can effectively prevent stenosis while maintaining proper stoma shape.

A

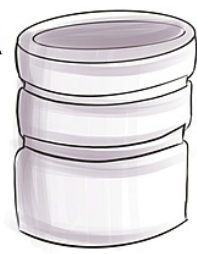

B

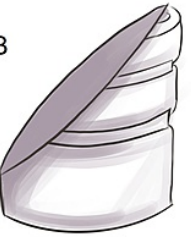

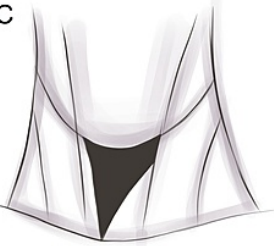
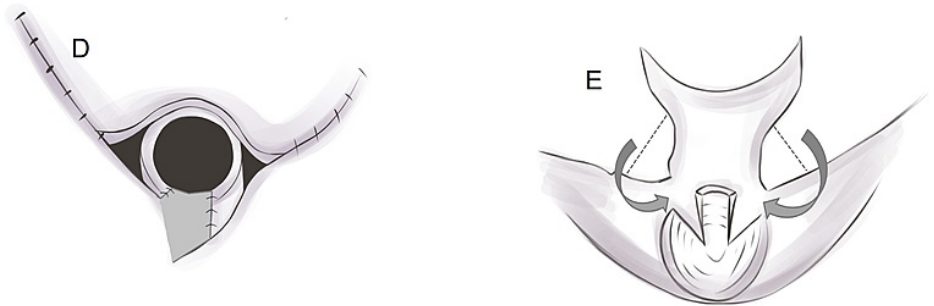

FIGURE 5: Schematic illustration of different stoma-fashioning techniques.

(A) Straight transection, (B) beveled transection, (C) wide triangular stoma, (D) interposed lower skin flap, and (E) Y-shaped, interposed superior skin flap.

The use of heat-and-moisture-exchange filters and hands-free speech valves requires a shallow and flat peristomal area for proper fitting of the adhesive patches [96]. An excessive stomal depth is undesirable for tracheoesophageal speech and tracheostoma care. Thus, division of the sternal heads of the 
sternocleidomastoid muscle is usually performed during a total laryngectomy to prevent recession and shrinkage of the tracheostoma [97].

\section{Conclusions}

Surgical techniques used in total laryngectomy are improving gradually. To remain relevant, several changes in operative procedures have been introduced to reduce complications and improve quality of life without compromising tumor control. These changes are partly driven by the increased complications of salvage total laryngectomy in the era of organ-preserving treatment. Moreover, the introduction of surgical voice rehabilitation can provide reasonable functional restoration and show a significant positive impact on the patient's quality of life. These are important issues that have recently gained attention.

Although many changes in total laryngectomy have been observed in recent decades, several conclusions related to benchmarking for surgical techniques cannot be reached. This situation can be primarily attributed to the lack of adequate and well-controlled studies, necessitating further studies. Thus, although total laryngectomy is one of the oldest cancer surgeries, issues related to the procedure remain open for discussion and will continue to require improvements in the near future.

\section{Additional Information \\ Disclosures}

Conflicts of interest: In compliance with the ICMJE uniform disclosure form, all authors declare the following: Payment/services info: All authors have declared that no financial support was received from any organization for the submitted work. Financial relationships: All authors have declared that they have no financial relationships at present or within the previous three years with any organizations that might have an interest in the submitted work. Other relationships: All authors have declared that there are no other relationships or activities that could appear to have influenced the submitted work.

\section{Acknowledgements}

The author would like to thank Dr Sombat Wongmanee (MD) and Ms. Benjamabhon Jittiworapan for their support in artworks and revision of the manuscript.

\section{References}

1. Matev B, Asenov A, Stoyanov GS, Nikiforova LT, Sapundzhiev NR: Losing one’s voice to save one’s life: a brief history of laryngectomy. Cureus. 2020, 12 :e8804. 10.7759/cureus.8804

2. Rzepakowska A, Żurek M, Niemczyk K: Review of recent treatment trends of laryngeal cancer in Poland: a population-based study. BMJ Open. 2021, 11:e045308. 10.1136/bmjopen-2020-045308

3. Richard JM, Sancho-Garnier H, Pessey JJ, et al.: Randomized trial of induction chemotherapy in larynx carcinoma. Oral Oncol. 1998, 34:224-228. 10.1016/s1368-8375(97)00090-0

4. Patel SA, Qureshi MM, Dyer MA, Jalisi S, Grillone G, Truong MT: Comparing surgical and nonsurgical larynx-preserving treatments with total laryngectomy for locally advanced laryngeal cancer. Cancer. 2019, 125:3367-77. 10.1002/cncr.32292

5. Wolf GT, Fisher SG, Hong WK, et al.: Induction chemotherapy plus radiation compared with surgery plus radiation in patients with advanced laryngeal cancer. N Engl J Med. 1991, 324:1685-90. 10.1056/NEJM199106133242402

6. Forastiere AA, Goepfert H, Maor M, et al.: Concurrent chemotherapy and radiotherapy for organ preservation in advanced laryngeal cancer. N Engl J Med. 2003, 349:2091-8. 10.1056/NEJMoa031317

7. NCCN clinical practice guidelines in oncology: head and neck cancers, version 3, 2021 . (2021). Accessed: September 7, 2021: https://www.nccn.org.

8. Bozec A, Culié D, Poissonnet G, Dassonville O: Current role of total laryngectomy in the era of organ preservation. Cancers (Basel). 2020, 12:10.3390/cancers12030584

9. Forastiere AA, Ismaila N, Lewin JS, et al.: Use of larynx-preservation strategies in the treatment of laryngeal cancer: American Society of Clinical Oncology clinical practice guideline update. J Clin Oncol. 2018, 36:1143-69. 10.1200/JCO.2017.75.7385

10. Weber RS, Berkey BA, Forastiere A, et al.: Outcome of salvage total laryngectomy following organ preservation therapy: the Radiation Therapy Oncology Group trial 91-11. Arch Otolaryngol Head Neck Surg. 2003, 129:44-9. 10.1001/archotol.129.1.44

11. Clark JH, Feng AL, Morton K, Agrawal N, Richmon JD: Neck incision planning for total laryngectomy with pharyngectomy. Otolaryngol Head Neck Surg. 2016, 154:650-6. 10.1177/0194599815621911

12. Sundaram K, Har-El G: The Wookey flap revisited. Head Neck. 2002, 24:395-400. 10.1002/hed.10035

13. Nigam A, Campbell JB, Dasgupta AR: Does the location of the laryngectomy stoma influence its ultimate size?. Clin Otolaryngol Allied Sci. 1993, 18:193-5. 10.1111/j.1365-2273.1993.tb00828.x

14. Timon CV, Toner M, Conlon BJ: Paratracheal lymph node involvement in advanced cancer of the larynx, hypopharynx, and cervical esophagus. Laryngoscope. 2003, 113:1595-9. 10.1097/00005537-20030900000035

15. Farlow JL, Birkeland AC, Rosko AJ, et al.: Elective paratracheal lymph node dissection in salvage laryngectomy. Ann Surg Oncol. 2019, 26:2542-8. 10.1245/s10434-019-07270-6

16. Khafif A, Yosef LM: Para-tracheal neck dissection - is dissection of the upper part of level VI necessary? . World J Otorhinolaryngol Head Neck Surg. 2020, 6:171-5. 10.1016/j.wjorl.2020.02.009 
17. de Bree R, Leemans CR, Silver CE, et al.: Paratracheal lymph node dissection in cancer of the larynx, hypopharynx, and cervical esophagus: the need for guidelines. Head Neck. 2011, 33:912-6. 10.1002/hed.21472

18. Joo YH, Sun DI, Cho KJ, Cho JH, Kim MS: The impact of paratracheal lymph node metastasis in squamous cell carcinoma of the hypopharynx. Eur Arch Otorhinolaryngol. 2010, 267:945-50. 10.1007/s00405-0091166-6

19. Lucioni M, D'Ascanio L, De Nardi E, Lionello M, Bertolin A, Rizzotto G: Management of paratracheal lymph nodes in laryngeal cancer with subglottic involvement. Head Neck. 2018, 40:24-33. 10.1002/hed.24905

20. Almulla A, Noel CW, Lu L, et al.: Radiologic-pathologic correlation of extranodal extension in patients with squamous cell carcinoma of the oral cavity: implications for future editions of the TNM classification. Int J Radiat Oncol Biol Phys. 2018, 102:698-708. 10.1016/j.ijrobp.2018.05.020

21. Sitges-Serra A, Lorente L, Mateu G, Sancho JJ: Therapy of endocrine disease: central neck dissection: a step forward in the treatment of papillary thyroid cancer. Eur J Endocrinol. 2015, 173:R199-206. 10.1530/EJE-150481

22. Li SX, Polacco MA, Gosselin BJ, Harrington LX, Titus AJ, Paydarfar JA: Management of the thyroid gland during laryngectomy. J Laryngol Otol. 2017, 131:740-4. 10.1017/S0022215117001244

23. Chang JW, Koh YW, Chung WY, Hong SW, Choi EC: Predictors of thyroid gland involvement in hypopharyngeal squamous cell carcinoma. Yonsei Med J. 2015, 56:812-8. 10.3349/ymj.2015.56.3.812

24. Mendelson AA, Al-Khatib TA, Julien M, Payne RJ, Black MJ, Hier MP: Thyroid gland management in total laryngectomy: meta-analysis and surgical recommendations. Otolaryngol Head Neck Surg. 2009, 140:298305. 10.1016/j.otohns.2008.10.031

25. Gaillardin L, Beutter P, Cottier JP, Arbion F, Morinière S: Thyroid gland invasion in laryngopharyngeal squamous cell carcinoma: prevalence, endoscopic and CT predictors. Eur Ann Otorhinolaryngol Head Neck Dis. 2012, 129:1-5. 10.1016/j.anorl.2011.04.002

26. McGuire JK, Viljoen G, Rocke J, Fitzpatrick S, Dalvie S, Fagan JJ: Does thyroid gland preserving total laryngectomy affect oncological control in laryngeal carcinoma?. Laryngoscope. 2020, 130:1465-9. 10.1002/lary.28235

27. Givi B, Linkov G, Ganly I, et al.: Selective neck dissection in node-positive squamous cell carcinoma of the head and neck. Otolaryngol Head Neck Surg. 2012, 147:707-15. 10.1177/0194599812444852

28. Ferlito A, Silver CE, Rinaldo A, Smith RV: Surgical treatment of the neck in cancer of the larynx . ORL J Otorhinolaryngol Relat Spec. 2000, 62:217-25. 10.1159/000027749

29. dos Santos CR, Gonçalves Filho J, Magrin J, Johnson LF, Ferlito A, Kowalski LP: Involvement of level I neck lymph nodes in advanced squamous carcinoma of the larynx. Ann Otol Rhinol Laryngol. 2001, 110:982-4. 10.1177/000348940111001016

30. Hassan OM, Mansour H, Metwaly O, Salah M: Evaluation of level I neck nodes involvement in advanced malignancy of the larynx and the hypopharynx. Egypt J Otolaryngol. 2021, 37:40. 10.1186/s43163-02100110-Z

31. Böttcher A, Betz CS, Bartels S, et al.: Rational surgical neck management in total laryngectomy for advanced stage laryngeal squamous cell carcinomas. J Cancer Res Clin Oncol. 2021, 147:549-59. 10.1007/s00432-02003352-1

32. Böttcher A, Olze H, Thieme N, et al.: A novel classification scheme for advanced laryngeal cancer midline involvement: implications for the contralateral neck. J Cancer Res Clin Oncol. 2017, 143:1605-12. 10.1007/s00432-017-2419-1

33. Sanabria A, Shah JP, Medina JE, et al.: Incidence of occult lymph node metastasis in primary larynx squamous cell carcinoma, by subsite, T classification and neck level: a systematic review. Cancers (Basel). 2020, 12:10.3390/cancers12041059

34. Paleri V, Urbano TG, Mehanna H, et al.: Management of neck metastases in head and neck cancer: United Kingdom National Multidisciplinary Guidelines. J Laryngol Otol. 2016, 130:S161-9. 10.1017/S002221511600058X

35. Shi Y, Zhou L, Tao L, Zhang M, Chen XL, Li C, Gong HL: Management of the N0 neck in patients with laryngeal squamous cell carcinoma. Acta Otolaryngol. 2019, 139:908-12. 10.1080/00016489.2019.1641219

36. Kim YH, Koo BS, Lim YC, Lee JS, Kim SH, Choi EC: Lymphatic metastases to level IIb in hypopharyngeal squamous cell carcinoma. Arch Otolaryngol Head Neck Surg. 2006, 132:1060-4.

10.1001/archotol.132.10.1060

37. Gross BC, Olsen SM, Lewis JE, Kasperbauer JL, Moore EJ, Olsen KD, Price DL: Level IIB lymph node metastasis in laryngeal and hypopharyngeal squamous cell carcinoma: single-institution case series and review of the literature. Laryngoscope. 2013, 123:3032-6. 10.1002/lary.24198

38. Jia S, Wang Y, He H, Xiang C: Incidence of level IIB lymph node metastasis in supraglottic laryngeal squamous cell carcinoma with clinically negative neck: a prospective study. Head Neck. 2013, 35:987-91. 10.1002/hed.23062

39. Graboyes EM, Zhan KY, Garrett-Mayer E, Lentsch EJ, Sharma AK, Day TA: Effect of postoperative radiotherapy on survival for surgically managed pT3N0 and pT4aN0 laryngeal cancer: analysis of the national cancer data base. Cancer. 2017, 123:2248-57. 10.1002/cncr.30586

40. Lim YC, Lee JS, Koo BS, Choi EC: Level IIb lymph node metastasis in laryngeal squamous cell carcinoma Laryngoscope. 2006, 116:268-72. 10.1097/01.mlg.0000197314.78549.d8

41. Furtado de Araújo Neto VJ, Cernea CR, Aparecido Dedivitis R, Furtado de Araújo Filho VJ, Fabiano Palazzo J, Garcia Brandão L: Cervical metastasis on level IV in laryngeal cancer . Acta Otorhinolaryngol Ital. 2014, 34:15-8.

42. Tu GY: Upper neck (level II) dissection for N0 neck supraglottic carcinoma . Laryngoscope. 1999, 109:467-70. 10.1097/00005537-199903000-00023

43. Ceachir O, Hainarosie R, Zainea V: Total laryngectomy - past, present, future . Maedica (Bucur). 2014, 9:2106.

44. Hinni ML, Ferlito A, Brandwein-Gensler MS, et al.: Surgical margins in head and neck cancer: a contemporary review. Head Neck. 2013, 35:1362-70. 10.1002/hed.23110 
45. Bradford CR, Wolf GT, Fisher SG, McClatchey KD: Prognostic importance of surgical margins in advanced laryngeal squamous carcinoma. Head Neck. 1996, 18:11-6. 10.1002/(SICI)10970347(199601/02)18:1<11::AID-HED2>3.0.CO;2-1

46. Zhang T, Cook I, Szczęśniak M, Maclean J, Wu P, Nguyen DD, Madill C: The relationship between biomechanics of pharyngoesophageal segment and tracheoesophageal phonation. Sci Rep. 2019, 9:9722. 10.1038/s41598-019-46223-7

47. Santoro GP, Maniaci A, Luparello P, Ferlito S, Cocuzza S: Dynamic study of oesophageal function during phonation: simple but effective. ORL J Otorhinolaryngol Relat Spec. 2021, 1-6. 10.1159/000513889

48. Öztürk K, Turhal G, Öztürk A, Kaya İ, Akyıldız S, Uluöz Ü: The comparative analysis of suture versus linear stapler pharyngeal closure in total laryngectomy: a prospective randomized study. Turk Arch Otorhinolaryngol. 2019, 57:166-70. 10.5152/tao.2019.4469

49. Galli J, Salvati A, Di Cintio G, Mastrapasqua RF, Parrilla C, Paludetti G, Almadori G: Stapler use in salvage total laryngectomy: a useful tool?. Laryngoscope. 2021, 131:E473-8. 10.1002/lary.28737

50. Dedivitis RA, Aires FT, Pfuetzenreiter EG Jr, Castro MA, Guimarães AV: Stapler suture of the pharynx after total laryngectomy. Acta Otorhinolaryngol Ital. 2014, 34:94-8.

51. Sannikorn P, Pornniwes N: Comparison of outcomes for staple and conventional closure of the pharynx following total laryngectomy. J Med Assoc Thai. 2013, 96 Suppl 3:S89-93.

52. Lee YC, Fang TJ, Kuo IC, Tsai YT, Hsin LJ: Stapler closure versus manual closure in total laryngectomy for laryngeal cancer: a systematic review and meta-analysis. Clin Otolaryngol. 2021, 46:692-8. 10.1111/coa.13702

53. Hui Y, Wei WI, Yuen PW, Lam LK, Ho WK: Primary closure of pharyngeal remnant after total laryngectomy and partial pharyngectomy: how much residual mucosa is sufficient?. Laryngoscope. 1996, 106:490-4. 10.1097/00005537-199604000-00018

54. Harris BN, Hoshal SG, Evangelista L, Kuhn M: Reconstruction technique following total laryngectomy affects swallowing outcomes. Laryngoscope Investig Otolaryngol. 2020, 5:703-7. 10.1002/lio2.430

55. Sweeny L, Golden JB, White HN, Magnuson JS, Carroll WR, Rosenthal EL: Incidence and outcomes of stricture formation postlaryngectomy. Otolaryngol Head Neck Surg. 2012, 146:395-402.

10.1177/0194599811430911

56. van der Putten L, Spasiano R, de Bree R, Bertino G, Leemans CR, Benazzo M: Flap reconstruction of the hypopharynx: a defect orientated approach. Acta Otorhinolaryngol Ital. 2012, 32:288-96.

57. Vasani SS, Youssef D, Lin C, Wellham A, Hodge R: Defining the low-risk salvage laryngectomy-A singlecenter retrospective analysis of pharyngocutaneous fistula. Laryngoscope Investig Otolaryngol. 2018, 3:11520. 10.1002/lio2.144

58. Gonzalez-Orús Álvarez-Morujo R, Martinez Pascual P, Tucciarone M, Fernández Fernández M, Souviron Encabo R, Martinez Guirado T: Salvage total laryngectomy: is a flap necessary? . Braz J Otorhinolaryngol. 2020, 86:228-36. 10.1016/j.bjorl.2018.11.007

59. Busoni M, Deganello A, Gallo O: Pharyngocutaneous fistula following total laryngectomy: analysis of risk factors, prognosis and treatment modalities. Acta Otorhinolaryngol Ital. 2015, 35:400-5. 10.14639/0392100X-626

60. Paleri V, Drinnan M, van den Brekel MW, et al.: Vascularized tissue to reduce fistula following salvage total laryngectomy: a systematic review. Laryngoscope. 2014, 124:1848-53. 10.1002/lary.24619

61. Chotipanich A, Wongmanee S: Management of deltopectoral flap failure using a three-stage revision reconstruction: a case report. J Surg Case Rep. 2021, 2021:rjab143. 10.1093/jscr/rjab143

62. Molteni G, Sacchetto A, Sacchetto L, Marchioni D: Optimal management of post-laryngectomy pharyngocutaneous fistula. Open Access Surg. 2020, 13:11-25. 10.2147/OAS.S198038

63. van der Kamp MF, Rinkel RN, Eerenstein SE: The influence of closure technique in total laryngectomy on the development of a pseudo-diverticulum and dysphagia. Eur Arch Otorhinolaryngol. 2017, 274:1967-73. 10.1007/s00405-016-4424-4

64. Thrasyvoulou G, Vlastarakos PV, Thrasyvoulou M, Sismanis A: Horizontal (vs. vertical) closure of the neopharynx is associated with superior postoperative swallowing after total laryngectomy. Ear Nose Throat J. 2018, 97:E31-5. 10.1177/0145561318097004-502

65. Oursin C, Pitzer G, Fournier P, Bongartz G, Steinbrich W: Anterior neopharyngeal pseudodiverticulum. A possible cause of dysphagia in laryngectomized patients. Clin Imaging. 1999, 23:15-18. 10.1016/s08997071(98)00032-1

66. Nitassi S, Belayachi J, Chihab M, et al.: Evaluation of post laryngectomy pharyngocutaneous fistula risk factors. Iran J Otorhinolaryngol. 2016, 28:141-7.

67. Deniz M, Ciftci Z, Gultekin E: Pharyngoesophageal suturing technique may decrease the incidence of pharyngocutaneous fistula following total laryngectomy. Surg Res Pract. 2015, 2015:363640. 10.1155/2015/363640

68. Walton B, Vellucci J, Patel PB, Jennings K, McCammon S, Underbrink MP: Post-Laryngectomy stricture and pharyngocutaneous fistula: review of techniques in primary pharyngeal reconstruction in laryngectomy. Clin Otolaryngol. 2018, 43:109-16. 10.1111/coa.12905

69. Aslıer NG, Doğan E, Aslıer M, İkiz AÖ: Pharyngocutaneous fistula after total laryngectomy: risk factors with emphasis on previous radiotherapy and heavy smoking. Turk Arch Otorhinolaryngol. 2016, 54:91-8. 10.5152/tao.2016.1878

70. Clavenna M, Obokhare J, Gill MT, Lian TS: Pharyngeal horizontal closure in total laryngectomies. Otolaryngol Head Neck Surg. 2012, 147:171-172. 10.1177/0194599812451426a148

71. Albirmawy OA, Elsheikh MN, Silver CE, Rinaldo A, Ferlito A: Contemporary review: impact of primary neopharyngoplasty on acoustic characteristics of alaryngeal tracheoesophageal voice. Laryngoscope. 2012, 122:299-306. 10.1002/lary.22459

72. Avci H, Karabulut B: Is it important which suturing technique used for pharyngeal mucosal closure in total laryngectomy? modified continuous connell suture may decrease pharyngocutaneous fistula. Ear Nose Throat J. 2020, 99:664-70. 10.1177/0145561320938918

73. Haksever M, Akduman D, Aslan S, Solmaz F, Ozmen S: Modified continuous mucosal connell suture for the 
pharyngeal closure after total laryngectomy: zipper suture. Clin Exp Otorhinolaryngol. 2015, 8:281-8. 10.3342/ceo.2015.8.3.281

74. Singer MI, Blom ED: Selective myotomy for voice restoration after total laryngectomy . Arch Otolaryngol. 1981, 107:670-3. 10.1001/archotol.1981.00790470018005

75. van Weissenbruch R, Kunnen M, Albers FW, van Cauwenberge PB, Sulter AM: Cineradiography of the pharyngoesophageal segment in postlaryngectomy patients. Ann Otol Rhinol Laryngol. 2000, 109:311-9. 10.1177/000348940010900314

76. Baugh RF, Lewin JS, Baker SR: Vocal rehabilitation of tracheoesophageal speech failures . Head Neck. 1990, 12:69-73. 10.1002/hed.2880120110

77. Blom ED, Pauloski BR, Hamaker RC: Functional outcome after surgery for prevention of pharyngospasms in tracheoesophageal speakers. Part I: speech characteristics. Laryngoscope. 1995, 105:1093-103. 10.1288/00005537-199510000-00016

78. Brok HA, Copper MP, Stroeve RJ, Ongerboer de Visser BW, Venker-van Haagen AJ, Schouwenburg PF: Evidence for recurrent laryngeal nerve contribution in motor innervation of the human cricopharyngeal muscle. Laryngoscope. 1999, 109:705-8. 10.1097/00005537-199905000-00005

79. Albirmawy OA, El-Guindy AS, Elsheikh MN, Saafan ME, Darwish ME: Effect of primary neopharyngeal repair on acoustic characteristics of tracheoesophageal voice after total laryngectomy. J Laryngol Otol. 2009, 123:426-33. 10.1017/S0022215108003861

80. Saha AK, Samaddar S, Choudhury A, Chaudhury A, Roy N: A comparative study of pharyngeal repair in two layers versus three layers, following total laryngectomy in carcinoma of larynx. Indian J Otolaryngol Head Neck Surg. 2017, 69:239-43. 10.1007/s12070-017-1108-3

81. Deschler DG, Doherty ET, Reed CG, Hayden RE, Singer MI: Prevention of pharyngoesophageal spasm after laryngectomy with a half-muscle closure technique. Ann Otol Rhinol Laryngol. 2000, 109:514-8. 10.1177/000348940010900513

82. Albirmawy OA: Effect of primary, cross-over, zigzag neopharyngoplasty on acoustic characteristics of alaryngeal, tracheoesophageal voice. J Laryngol Otol. 2011, 125:841-8. 10.1017/S0022215111000910

83. Horowitz JB, Sasaki CT: Effect of cricopharyngeus myotomy on postlaryngectomy pharyngeal contraction pressures. Laryngoscope. 1993, 103:138-40. 10.1002/lary.5541030203

84. Op de Coul BM, van den Hoogen FJ, van As CJ, Marres HA, Joosten FB, Manni JJ, Hilgers FJ: Evaluation of the effects of primary myotomy in total laryngectomy on the neoglottis with the use of quantitative videofluoroscopy. Arch Otolaryngol Head Neck Surg. 2003, 129:1000-5. 10.1001/archotol.129.9.1000

85. Kaye R, Tang CG, Sinclair CF: The electrolarynx: voice restoration after total laryngectomy . Med Devices (Auckl). 2017, 10:133-40. 10.2147/MDER.S133225

86. Lorenz KJ: Rehabilitation after total laryngectomy: a tribute to the pioneers of voice restoration in the last two centuries. Front Med (Lausanne). 2017, 4:81. 10.3389/fmed.2017.00081

87. Jassar P, England RJ, Stafford ND: Restoration of voice after laryngectomy. J R Soc Med. 1999, 92:299-302. 10.1177/014107689909200608

88. Brook I, Goodman JF: Tracheoesophageal voice prosthesis use and maintenance in laryngectomees . Int Arch Otorhinolaryngol. 2020, 24:e535-8. 10.1055/s-0039-3402497

89. Luu K, Chang BA, Valenzuela D, Anderson D: Primary versus secondary tracheoesophageal puncture for voice rehabilitation in laryngectomy patients: a systematic review. Clin Otolaryngol. 2018, 43:1250-9. 10.1111/coa.13138

90. Barauna Neto JC, Dedivitis RA, Aires FT, Pfann RZ, Matos LL, Cernea CR: Comparison between primary and secondary tracheoesophageal puncture prosthesis: a systematic review. ORL J Otorhinolaryngol Relat Spec. 2017, 79:222-9. 10.1159/000477970

91. Paleri V, Wight RG, Owen S, Hurren A, Stafford FW: Defining the stenotic post-laryngectomy tracheostoma and its impact on the quality of life in laryngectomees: development and validation of a stoma function questionnaire. Clin Otolaryngol. 2006, 31:418-24. 10.1111/j.1749-4486.2006.01287.x

92. De Virgilio A, Greco A, Gallo A, Martellucci S, Conte M, de Vincentiis M: Tracheostomal stenosis clinical risk factors in patients who have undergone total laryngectomy and adjuvant radiotherapy. Eur Arch Otorhinolaryngol. 2013, 270:3187-9. 10.1007/s00405-013-2695-6

93. Wax MK, Touma BJ, Ramadan HH: Tracheostomal stenosis after laryngectomy: incidence and predisposing factors. Otolaryngol Head Neck Surg. 1995, 113:242-7. 10.1016/S0194-5998(95)70112-5

94. Suzuki M, Tsunoda A, Shirakura S, Sumi T, Nishijima W, Kishimoto S: A novel permanent tracheostomy technique for prevention of stomal stenosis (triangular tracheostomy). Auris Nasus Larynx. 2010, 37:465-8. 10.1016/j.anl.2009.11.007

95. Isshiki N, Tanabe M: A simple technique to prevent stenosis of the tracheostoma after total laryngectomy . J Laryngol Otol. 1980, 94:637-42. 10.1017/s0022215100089349

96. van der Houwen EB, van Kalkeren TA, Post WJ, Hilgers FJ, van der Laan BF, Verkerke GJ: Does the patch fit the stoma? A study on peristoma geometry and patch use in laryngectomized patients. Clin Otolaryngol. 2011, 36:235-41. 10.1111/j.1749-4486.2011.02307.x

97. Santoro GP, Luparello P, Lazio MS, Comini LV, Martelli F, Cannavicci A: Myotomy of sternocleidomastoid muscle as a secondary procedure in laryngectomized patients. Head Neck. 2019, 41:3743-6. 10.1002/hed.25852 